\title{
A Review of Biomimetic Nanoparticle Drug Delivery Systems Based on Cell Membranes
}

This article was published in the following Dove Press journal:

Drug Design, Development and Therapy

\author{
Meilin Zhang $\mathbb{D}^{\prime}$ \\ Ying Du' \\ Shujun Wang ${ }^{2}$ \\ Baoan Chen'
}

'Department of Hematology and Oncology, Zhongda Hospital, School of Medicine, Southeast University, Nanjing, Jiangsu Province 210009, People's Republic of China; ${ }^{2}$ Jinling Hospital Department of Blood Transfusion, School of Medicine, Nanjing University, Nanjing, Jiangsu Province 210002, People's Republic of China
Correspondence: Baoan Chen Department of Hematology and Oncology, Zhongda Hospital, School of Medicine, Southeast University, Dingjiaqiao 87, Gulou District, Nanjing, Jiangsu Province 210009, People's Republic of China

Tel +862583272006

Fax +862583272011

Email cba8888@hotmail.com

\begin{abstract}
Cancers have always been an intractable problem because of recurrence and drug resistance. In the past few decades, nanoparticles have been explored intensely to diagnose, prevent and treat malignancy due to their good penetrability and better targeting. However, most nanocarriers have poor biodegradation and can be discharged out of the body quickly or cleared by immune cells while failing to obtain effective drug concentration at the specific sites. The emergence of biological membrane encapsulation technology relieves the fast clearance of antitumor drugs and reduces toxicity in vivo. This review will discuss the advantages and disadvantages of several blood cell membrane-coated nanoparticles and further introduce exosome-carried drugs to evidence the promising prospect of biomimetic nanoparticle drug delivery systems.
\end{abstract}

Keywords: tumor, drug delivery systems, blood cells, biological membranes

\section{Introduction}

Recently, the number of people who are suffering from cancer increased rapidly due to environment pollution and unhealthy lifestyle. It has always been an alarming problem for the past several decades around the world. Although the treatment of malignant tumor has developed rapidly, traditional chemotherapy and phototherapy are still the most common methods. However, common chemotherapeutic agents have a few fatal drawbacks in clinical application, such as poor water solubility, low oral bioavailability and serious adverse reactions over therapeutic effect. ${ }^{1}$ In addition, a series of new problems arise from single or combined therapy, such as recurrence, drug resistance, systemic side effects and so on.

Nontargeted drugs affect the normal cells or organs instead of the disease location. Therefore, the development of suitable carriers that can improve the therapeutic efficacy at specific sites and reduce drug resistance by increasing the accumulation of chemotherapy drugs at the targeted sites to achieve high targeted efficiency and low general toxicity becomes an indispensable need. Under this circumstance, the application of molecular targeted drugs has caught scientists' attention and they have made a great breakthrough. Recent studies showed that nanoparticles structured smaller than $100 \mathrm{~nm}$ in at least one dimension had a tremendous potential as drug carriers due to their small size and unique physicochemical and biological properties that make them an appealing material for biomedical applications. ${ }^{2}$ Nanoparticle drug delivery systems are widly researched in medical treatment, including a large variety of forms like liposomes, polymer nanoparticles, solid lipid nanoparticles, hybrid nanoparticles, biomimetic nanoparticles, and so on. ${ }^{3}$ Compared with traditional 
treatments, nanoscale drug carriers have the advantages of good penetrability, strong targeting, and long-acting time. Nanoparticles are continuously engineered and perform the potential to transform the diagnosis, prevention and treatment of difficult-to-treat diseases such as cancer, Alzheimer's disease, stroke, and diabetes. ${ }^{4-6}$ However, the development of functional nanoparticles is still limited by its nature. Liposomes, metal nanoparticles and polymer nanomaterials are the most commonly used nanocarriers. While most liposomes can only carry hydrophilic drugs and be discharged out of the body quickly the latter two have poor biodegradation as a result that they are easily retained in liver and kidney tissues to cause toxic damage, and it is difficult to obtain effective drug concentration at the specific sites.

Under the circumstances, biological drug delivery systems such as albumin, erythrocytes, and platelets with the advantages of nontoxic and immunogenicity, innate biocompatibility, large drug loading, and high targeting have come into being and have been proposed to play an important role in tumor treatment. This article reviews some biocompatible and biodegradable nanoparticles used in cancer administration, which includes not only biological membrane like hemocyte, but also some synthetic polymeric nanoparticles (Table 1), from the best synthetic polymeric nanoparticles PLGA to the combination of nature cell membranes NPs, to achieve a feasible precision medicine for tumor therapeutic applications.

\section{PLGA-based Nanoparticles}

Poly (lactic-co-glycolic acid) (PLGA) has been one of the most studied polymeric carriers approved by the FDA for anticancer therapy because of its low systemic toxicity and high biodegradability compared to other polymeric systems. ${ }^{7}$ As a controlled-release formulation of drugs, ${ }^{8}$ drug molecules in NPs are slowly released from the pores of the material surface as the PLGA degrades, as a result, the drugs maintain a constant concentration for a long time, prolong the exposure time, and improve the bioavailability. ${ }^{9}$ What is more, another attractive characteristic of PLGA over other nanoparticles is

Table I Examples of Nanoparticles and Therapeutic Moieties Loaded by Erythrocyte/Platelet/Leukocyte/Exosome in Various vitro and vivo Tumor Models

\begin{tabular}{|c|c|c|c|c|}
\hline $\begin{array}{l}\text { Coated } \\
\text { Membranes }\end{array}$ & Inner Cores & Therapeutic Moieties & Applications & References \\
\hline \multirow[t]{4}{*}{ Erythrocyte } & DSPE-PEG & Paclitaxel & Chemotherapy for gastric cancer & 29 \\
\hline & CuS & Doxorubicin & Synergistic photo-chemotherapy for melanoma & 30 \\
\hline & - & $\begin{array}{l}\text { I,2-diaminocyclohexane- } \\
\text { platinum (II) and ICG }\end{array}$ & Synergistic photo-chemotherapy & 31 \\
\hline & PCEC & IR780 iodide and docetaxel & Photo-chemotherapy for breast cancer & 32 \\
\hline \multirow[t]{6}{*}{ Platelet } & PLGA & MRI contrast agent & Detection of atherosclerosis & 4 \\
\hline & PLGA & Bufalin & Chemotherapy for hepatic carcinoma & 23 \\
\hline & PLGA & Docetaxel & Coronary restenosis & 35 \\
\hline & - & Adriamycin & Chemotherapy for lymphoma & 36 \\
\hline & Gold nanorods & - & Photothermal therapy for squamous cell carcinoma & 37 \\
\hline & $\mathrm{ZrO} 2 \mathrm{NPs}$ & & Cervical cancer & 38 \\
\hline \multirow[t]{2}{*}{ Leukocyte } & PLGA & Doxorubicin & Glioma & 40 \\
\hline & Silica nanoparticles & Doxorubicin & Breast cancer & 41 \\
\hline \multirow[t]{3}{*}{ Exosome } & Silicon nanoparticles & Doxorubicin & $\begin{array}{l}\text { Chemotherapy for hepatocarcinoma, breast cancer, } \\
\text { lung metastasis tumor }\end{array}$ & 45 \\
\hline & $\begin{array}{l}\text { Copper-cysteamine } \\
\text { nanoparticles }\end{array}$ & - & Photothermal therapy for hepatocarcinoma & 51 \\
\hline & - & Doxorubicin & Chemotherapeutic for osteosarcoma & 52 \\
\hline
\end{tabular}


that PLGA is one of the best characterized biodegradable copolymers because it decomposes into nontoxic lactic acid and glycolic acid and further into $\mathrm{H}_{2} \mathrm{O}$ and $\mathrm{CO}_{2}$ eliminated from the body with low to no toxicity. ${ }^{10,11}$ According to all these advantages of PLGA NPs, are extensively used in a variety of fields, for example, PLGA NPs loaded with corresponding therapeutic drugs can cross the blood-brain barrier and reach the central nervous system. ${ }^{12}$

In addition to the intrinsic properties of PLGA NPs, incorporating targeting ligands, such as small molecules, peptides, antibodies, and aptamers onto their surface make them better with respect to targeting strategy, biocompatibility, and preferential accumulation. ${ }^{10,13}$ Zheng et al verified co-delivery of sorafenib and metapristone by the LFC131-conjugated (a peptide inhibitor of CXCR4) PLGA NPs showed prolonged circulation and target accumulation at tumor sites, and thus suppressed tumor growth in HCC models. ${ }^{14}$ Byeon et al developed HA-labeled PLGA NPs (HA-PLGA NPs) incorporating both PTX and FAK siRNA as a two-in one delivery system to increase the efficiency of targeted delivery against tumor-specific receptors and to enhance therapeutic efficacy in drug-resistant ovarian cancer. $^{15}$

In comparison to monotherapy, anticancer drugs encapsulated in PLGA nanoparticles show good biocompatibility, strong cytotoxicity, significant antitumor activity and minimal systemic toxicity. ${ }^{11,15}$ Co-encapsulation of two drugs to the tumor via single nanoparticles could better maintain the synergistic drug effect in vivo than the coadministration of two single drugs and drug-carried nanoparticles. ${ }^{16}$ What is more, as a kind of biodegradable organic polymer, PLGA has good modifiability.

\section{Biofilm Nanoparticles}

Cell membrane camouflaged nanoparticles have been exploited as drug-delivery carriers for several decades because of the improvement of the targeting efficiency and retention of carried drugs. Originally, the initial cell membrane-based nanoparticles (CMBNPs) were fabricated from a red blood cell membrane shell and PLGA core through a co-extrusion process, forming a core-shell structure. ${ }^{17}$ Subsequently, all kinds of cell membranes, such as platelets, albumin, cancer cells, and exosome vesicles, have been exploited as carriers to carry various drugs in different fields. ${ }^{18-21}$ There were three basic strategies for the development of biomimetic nanoparticles: (1) synthetic NPs modified with targeting ligands that mimic cell surface proteins, (2) cell membrane-coated
NPs, and (3) liposomes engineered with cell membrane proteins. $^{22}$

Though the retention time of drug improves after PEGmodified NPs, the immune response to synthetic materials still concerns researchers, as well as that the most synthetic nanoparticles have poor bioavailability which lead to low accumulation and quick excretion in vivo ${ }^{13}$. Furthermore, simple surface functionalization methods cannot accurately simulate the complex interfaces that exist in nature, and finally NPs will be recognized as foreign bodies and cannot completely escape subsequent immune response. ${ }^{23}$ Under this circumstance, scientists turn their interest to natural cells, which are the fundamental units in biosome. ${ }^{24}$ Cells perform specific and basic tasks through different significant biomacromolecules, which are embedded into cell membranes, a lipid bilayer equipped with a collection of biomacromolecules. ${ }^{25}$ Cell membranes are able to control the physical interactions between each other, like regulating immune functions, receiving signals, and anchoring binding interactions. ${ }^{25,26}$ Take erythrocytes as an example, nonnuclear, abundant, long half-life (up to 120 days in blood circulation), all these key advantages made RBC membrane-coated NPs the hottest carrier to deliver various chemotherapeutics. ${ }^{27}$

Cell membrane coating retains the complex composition of the entire cell surface on the nanoparticle, bypassing the necessary for complicated chemistry and the identification of individual membrane components. Blood cells are the most used membranes to envelop NPs, (Figure 1), along with a popular new-born carrier, exosomes. On account of the combined advantages of both synthetic nanoparticle cores and natural cell membranes, biofilm nanoparticles have gained increasing attention in precise molecular drug delivery in cancer. $^{3}$

\section{Erythrocyte Membranes Coated NPs (RBCNPs)}

In the realm of tumor diagnosis and therapy, nanoparticles have revealed promising achievements with high penetration and targeting. But the short drug accumulation time, low dose in tumor tissues, and immune elimination as a foreign body are subsequent problems. Under the circumstance, the blood membrane enveloped nanoparticles emerge.

As the first studied drug coating biological membrane, the research of red blood cells as nanofilms has a long history. Erythrocytes are the most numerous blood cell up 


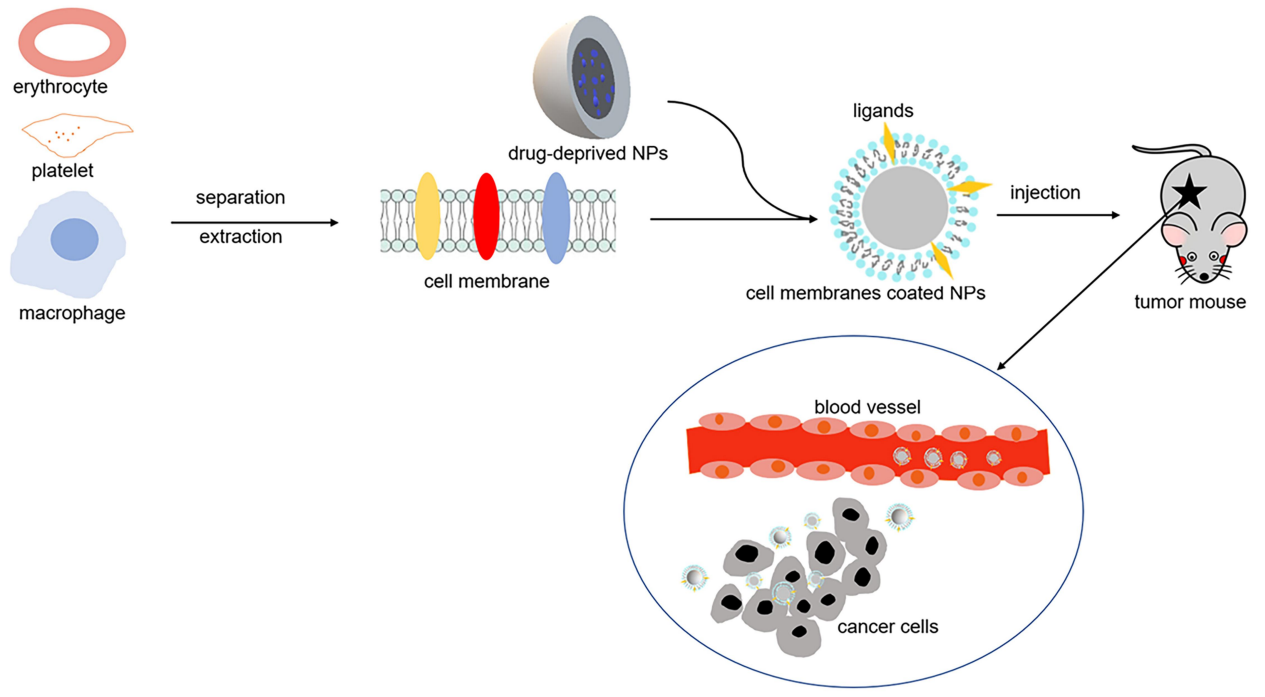

Figure I Schematic illustration of erythrocyte/platelet/macrophage membrane-coated nanoparticles for tumor therapy in tumor-bearing mice.

to fvie billion per milliliter in human circulation. ${ }^{28}$ This feature guarantees a huge material bank package for nanodrugs. Furthermore, without nucleus and organelles, mature RBC membranes are advantageous for extraction and purification. The unique double concave discoid shape provides RBCs a larger superficial area to carry other cargoes, such as oxygen and functional proteins. It has been proved that combining associated proteins and other elements membranes enhanced the targeting and penetration capabilities of core anticancer drugs. ${ }^{29}$ Meanwhile, drug accumulation intensity in tumor is much stronger than that of normal tissues in RBCNPs when contrasted with control groups, and thus showing better inhibition of tumor growth. ${ }^{30}$

$\mathrm{CD} 47$, as the self-marker protein on RBC surfaces, interact with signal-regulatory protein $\alpha(\operatorname{SIRP} \alpha)$ expressed by macrophages through sending signal "no eating me". Finally, RBCNPs also improve the immune-evading capability and effectually avoid uptake by immune cells when compared with bare NPs. ${ }^{31}$ Last but not least, erythrocytes have a much longer life span for about 120 days in circulation. In comparison to synthetic encapsulation, nanoparticles encapsulated with RBC membranes relatively possess longer circulation and retention time. ${ }^{32}$

In other words, huge quantity, unique construction, long lifespan, along with escaping the immune system, contribute to the particular superiority of an erythrocytecoated drug delivery system, which also accounts for the hot extensive research into erythrocytes camouflaged nanoparticles.

\section{Platelet Membranes Coated NPs (PNPs)}

As a natural ingredient in blood circulation, platelets are nuclear-free fragments generated by megakaryocytes in bone marrow in adults. Like erythrocytes they are nonnuclear and their small size enables them to be extracted relatively easily. Due to more receptors on the surface, platelets show more complexity and multifactorial functionality. They are able to avoid phagocytic uptake by macrophages. Platelets exist in lots of physiological and pathological processes such as wound healing, inflammatory reaction, thrombosis, and organ transplantation rejection. Studies show that they also play an important role in tumors as a result of the interaction with many receptors in tumor cell membranes, ${ }^{33}$ for example, platelets participate in tumor metastasis and neovascularization, moreover, tumor cells induce platelet is aggregation which known as TCIPA. Platelets have membrane protein P-selectin to mediate the binding of platelets to CD44 on tumor cells, ${ }^{34}$ which endows PNPs particular functionality to CD44 high expression tumors.

Owing to the advantages of natural affinity to tumor cells, platelet membranes have inspired the design of many functional nanocarriers. In the area of tumor targeted therapy, researchers demonstrated that anticancer drugs could be incorporated into nano-polymeric cores, and these drug-loaded nanoparticles manifested considerable efficacy in tumor mouse models. ${ }^{23} \mathrm{Hu}$ et al adopted a cell membrane cloaking technique to prepare PNPs, whose final size were slightly larger about $15 \mathrm{~nm}$ than the bare NPs. And compared to bare NPs and RBCNPs, the platelets coated NPs showed 
significantly enhanced permeation and retention effect in vivo. ${ }^{35}$ Coating with natural platelet membrane, PNPs are capable of navigating through circulation and distributing to diseased tissue preferentially rather than healthy position. ${ }^{4} \mathrm{Xu}$ et al used platelets to transport doxorubicin (DOX) to treat lymphoma and they found DOX-loaded with platelets targeted cancer cells well through the phenomenon "TCIPA" (Figure 2), and the growth inhibition of cancer cells was strengthened while the cardiotoxicity of DOX was weakened. ${ }^{36}$ In addition, Rao et al combined photothermal therapy with tumor cells targeted platelets delivery system, which could subsequently damage the tumor tissues and accumulate the PNPs. ${ }^{37}$ Compared to uncoated particles, the platelet membrane-cloaked nanoparticles have reduced cellular uptake by macrophage-like cells, thus reducing their clearance and extending the circulation time. ${ }^{35,38}$ Introducing additional elements onto the outer surface of NPs to add extra functionality via polymer tethers enable anticancer drug to be most absorbed and utilized by targeted tissues, and further improve the common advantages of platelets and traditional synthetic nanoparticles.

All results showed that drug-loaded PNPs gave an efficacious chemotherapeutic treatment and effectively inhibited the growth of carcinoma, presenting the unique self-reinforcing characteristic of this delivery system in the treatment of cancer.

\section{Leukocyte Membranes Coated NPs (LNPs)}

Leukocytes (white blood cells), including granulocytes, lymphocytes and monocytes, which belong to the immune system, have larger volumes than RBCs because of their nucleus. Inflammation, which is regulated by WBCs, is closely related to the tumor microenvironment. The leukocytes participated in neovascularization, metastasis and progress of tumor through the interaction with all kinds of cytokines, chemokines and proteins between tumor cells, such as TNF- $\alpha$, RANKL which is called leukocyte infiltration. ${ }^{39}$

Macrophages have drawn the most attention among all the leukocytes to be designed as the coats of nanoparticles in recent years. ${ }^{40}$ Coated with macrophage membranes, NPs own the ability to easily migrate between blood vessels and other extravascular tissues as well as macrophages. Besides, macrophages naturally phagocytose malignant cells and decrease fibrosis. It has been proved that compared to conventional NPs, the macrophage membrane-coated ones (MNPs) can effectively transport anticancer drugs to specific sites and achieve prominent accumulation time with a controlled release effect. ${ }^{41}$ The WBC membranes also act against opsonization and reticuloendothelial system (RES) clearance which serves as a tumor-homing navigator to enhance tumor accumulation. ${ }^{42}$ As a biofilm nanoparticle, macrophage derived NPs reveal excellent biocompatibility and immune evasion ability via CD47 embedded in membranes.

However, imperfectly as karyocytes, compared with RBCs and platelets, the extraction and purification of leukocytes are more complex. In addition, the main histocompatibility complex (MHC) molecules expressed on its

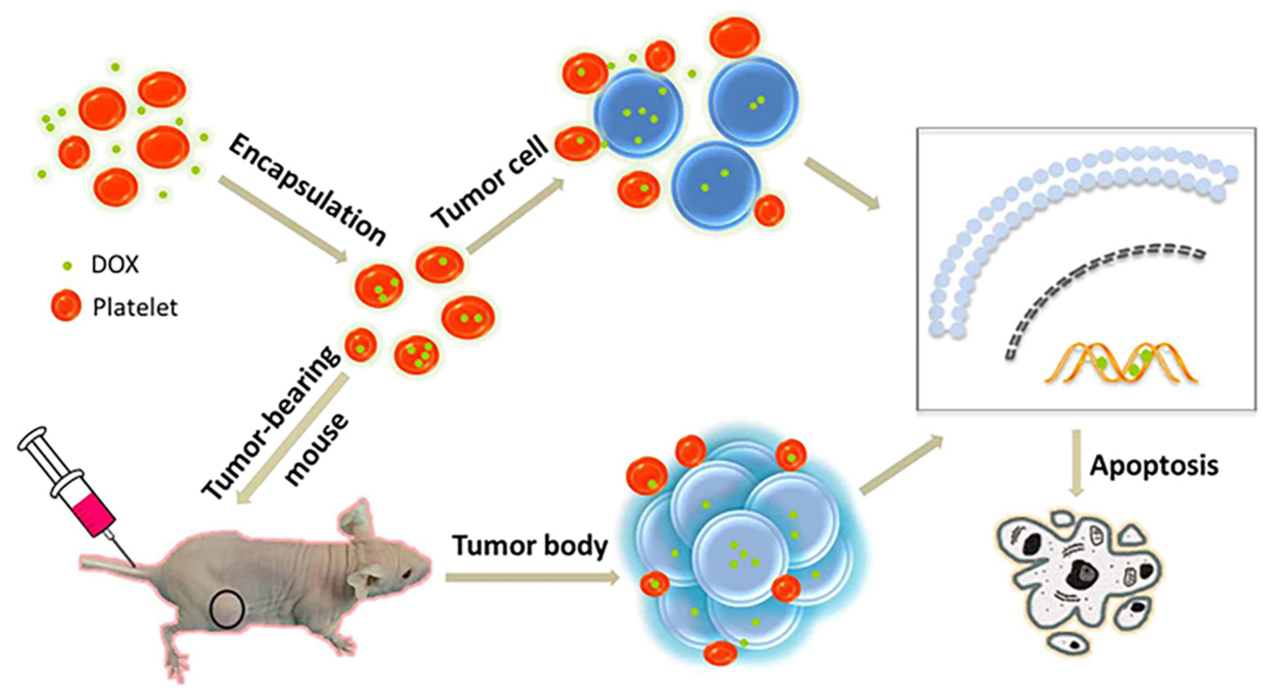

Figure 2 Schematic illustration of the mechanism of enhanced anticancer activity of DOX-platelet through "tumor cell-induced platelet aggregation". Note: Reproduced from Xu P, Zuo H, Chen B, et al. Doxorubicin-loaded platelets as a smart drug delivery system: an improved therapy for lymphoma. Sci Rep. 2017;7:42632. Creative Commons License and Disclaimer Available from: http://creativecommons.org/licenses/by/4.0/legalcode. ${ }^{36}$ 
exterior may lead to incompatibility. Irrespective of its shortcoming, the targeting and regulating ability on tumor and inflammation of WBC make it a promising candidate for the encapsulation of NPs.

\section{Exosomes Based NPs}

Exosomes are nanosized (30-120 nm) membrane vesicles excreted by various types of cells which are acting as a mean of long-distance intercellular communication and biomaterial transfers without cell-to-cell contact. ${ }^{25,43}$ What cannot be ignored is that exosomes originated from inward budding of membrane of multivesicular bodies and are released from the cells into the extracellular environment with the plasma membrane $^{44}$ (Figure 3), therefore, a broad kind of cargo on cell membranes of the parent cells are contained in exosomes, including nucleic acids, proteins and enzymes. ${ }^{45}$ Researchers demonstrate that exosomes are associated with several hallmark features of cancers, including stimulating tumor angiogenesis, rebuilding the stroma to establish the tumor microenvironment, as well as facilitating tumor growth and drug resistance by the means of horizontal transfer of genetic information between cancer cells. ${ }^{46}$
The therapeutic application of exosomes is promising because injected exosomes are effectively entering other cells with minimal immune clearance and repeated injections are well tolerated without substantial side effects. ${ }^{44,47,48}$ It is worth noting that exosomes are enriched with transmembrane protein and adhesion proteins, and also specific protein receptors, therefore, protein cargoes on exosomes can be transported to and receipted by targeted cells effectively to induce cellular responses, such as the inducing and promoting of neoplasia. ${ }^{49}$ In addition, exosomes present an excellent capacity to escape the clearance by immune system depending on protein CD47, which is a widely expressed integrin associated transmembrane protein that functions in part to protect cells from phagocytosis. ${ }^{50}$ Inspired from these advantages, exosomes are engineered as effective vehicles for therapeutics. Huang et al found that in photodynamic therapy, the soluble nanodrugs were able to enter exosomes secreted by tumor cells, and supposed that exosomes could be used to deliver drugs to target pancreatic cells. ${ }^{51}$

Of particular concern, additional characters of exosomes in delivering functional cargos to diseased cells also favor their use as drug deliveries. The lipid and

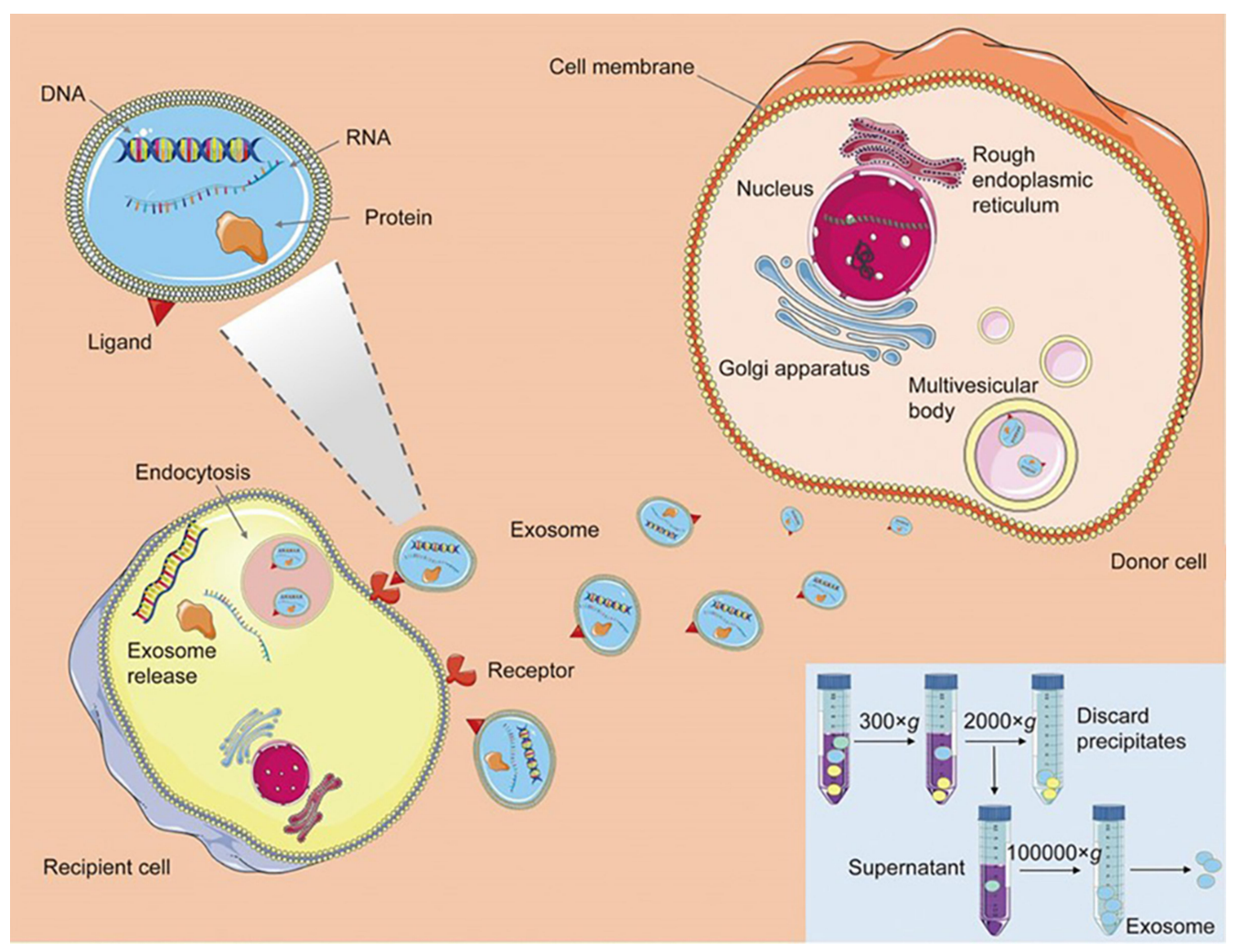

Figure 3 Schematic illustration of generation, release and cargo transfer of exosomes.

Note: Reprinted by permission from Springer Nature. Acta Pharmacologica Sinica. Engineering exosomes as refined biological nanoplatforms for drug delivery. Luan X, Sansanaphongpricha K, Myers I, Chen H, Yuan H, Sun D. 2017;38(6):754-763. Copyright (C 2017. ${ }^{20}$ 
protein composition and other natural constituents of exosomes play a role in enhanced bioavailability and in minimizing adverse reactions, suggesting that biocompatibility is guaranteed and systemic toxicity does not need to be taken into consideration when compared with artificial chemical delivery vehicles. By comparison with free chemotherapeutics, the exosome-loaded ones can kill tumor cells more effectively and show low cytotoxicity in myocardial cells and other healthy tissues. ${ }^{52}$ Once nanoparticle-loaded exosomes are generated, they will be preferentially captured by the same cell line. Therefore, specific exosome-based transports are preferentially transferred to typical cell types but not to others. ${ }^{53}$

While as a distinct type of nanocarrier, already carrying numerous proteins and nucleic acids by their nature, exosomes relatively fail to achieve high loading capacity. ${ }^{43}$ Moreover, the successful modification, purification and analytical procedures require more exploration. ${ }^{20,54}$ In general, exosomes are extracellular membrane-derived vesicles derived from most cell types, which can be engineered to deliver diverse therapeutic payloads as worthwhile nanocarriers.

\section{Conclusion}

With the advantages of harmless degradation products, synthetic PLGA NPs have been functionalized with targeted peptides or other functional groups to increase the capacity of targeting delivery of anticancer drugs to tumors. And co-delivery of multiple drugs in a single nanocarrier shows a better synergistic drug effect and lower side effects than simple combined administration, thus the recurrence and drug resistance rates drop considerably.

However, there are still a few drawbacks limiting synthetic NPs to meet clinical expectation used on patients. As a drug delivery system, nanoparticles are transported to their target tissues via blood environment, thus the compatibility of engineered NPs with blood constituents must be taken into consideration with extreme care. Scientists found that PLGA NPs could result in the damage and premature elimination of erythrocytes because they accelerate the clearance of blood cells in the way of the alteration of cells' functionality, mechanical properties and integrity. ${ }^{6}$ Blood cell membrane-coated nanoparticles have the capability to overcome these problems like immune elimination. Both in vitro and vivo, cell membranecoated NPs have significantly enhanced the retention of drugs and retained cytotoxicity toward carcinoma. In fact, natural biofilm-based nanoparticles are equipped with the capability to unite advantages of both synthetic nanocarriers and cellmediated drug delivery. They possess low immunogenicity which is able to avoid the rapid clearance and low toxicity to effectively spare healthy cells when compared with nonnatural vehicles. Biofilm nanoparticles display prolonged circulation and preferential targeting to disease tissues which enhance tumor-targeting property of chemotherapeutics to malignant tumors. Although the development of nanomedicine has been studied for a long time, few clinical applications are available. The request of efficient and safety materials to deliver drugs and the potential additional risk on human health are challenges demanding prompt solution.

In conclusion, with the rapid development of encapsulation skills, bionanoparticles that combine the unique functionalities of natural biomaterials, such as cell membranes or membrane vesicles have recently increased considerable attention as effective drug delivery platforms.

\section{Acknowledgments}

This work was supported by the Jiangsu Social Development Project (Project No. BE 2018711), Southeast University Project (Project No. 2018yy-jccx003), the Key Medical Projects of Jiangsu Province (BL2014078), the Key Department of Jiangsu Province (2016-2020).

\section{Disclosure}

The authors report no conflicts of interest in this work.

\section{References}

1. Li S, Li X, Ding J, Han L, Guo X. Anti-tumor efficacy of folate modified PLGA-based nanoparticles for the co-delivery of drugs in ovarian cancer. Drug Des Devel Ther. 2019;13:1271-1280. doi:10.2147/DDDT.S195493

2. Wilczewska AZ, Niemirowicz K, Markiewicz KH, Car H. Nanoparticles as drug delivery systems. Pharmacol Rep. 2012;64(5):1020-1037.

3. Jin K, Luo Z, Zhang B, Pang Z. Biomimetic nanoparticles for inflammation targeting. Acta Pharm Sin B. 2018;8(1):23-33.

4. Wei X, Ying M, Dehaini D, et al. Nanoparticle functionalization with platelet membrane enables multifactored biological targeting and detection of atherosclerosis. ACS Nano. 2018;12(1):109-116. doi:10.1021/acsnano.7b07720

5. Rong X, Ji Y, Zhu X, et al. Neuroprotective effect of insulin-loaded chitosan nanoparticles/PLGA-PEG-PLGA hydrogel on diabetic retinopathy in rats. Int J Nanomedicine. 2019;14:45-55. doi:10.2147/IJN.S184574

6. de la Harpe KM, Kondiah PPD, Choonara YE, Marimuthu T, Du Toit LC, Pillay V. The hemocompatibility of nanoparticles: a review of cell-nanoparticle interactions and hemostasis. Cells. 2019;8 (10):1209. doi:10.3390/cells8101209

7. Surendran SP, Moon MJ, Park R, Jeong YY. Bioactive nanoparticles for cancer immunotherapy. Int J Mol Sci. 2018;19:12. doi:10.3390/ijms19123877

8. Zhong Y, Su T, Shi Q, et al. Co-administration of iRGD enhances tumor-targeted delivery and anti-tumor effects of paclitaxel-loaded PLGA nanoparticles for colorectal cancer treatment. Int J Nanomedicine. 2019;14:8543-8560. doi:10.2147/IJN.S219820

9. Duan T, Xu Z, Sun F, et al. HPA aptamer functionalized paclitaxel-loaded PLGA nanoparticles for enhanced anticancer therapy through targeted effects and microenvironment modulation. Biomed Pharmacother. 2019;117:109121. doi:10.1016/j.biopha.2019.109121 
10. Rezvantalab S, Drude NI, Moraveji MK, et al. PLGA-based nanoparticles in cancer treatment. Front Pharmacol. 2018;9:1260. doi:10.3389/fphar.2018.01260

11. Gao J, Liu J, Xie F, Lu Y, Yin C, Shen X. Co-delivery of docetaxel and salinomycin to target both breast cancer cells and stem cells by PLGA/TPGS nanoparticles. Int $J$ Nanomedicine. 2019;14:9199-9216. doi:10.2147/IJN.S230376

12. Rigon L, Salvalaio M, Pederzoli F, et al. Targeting brain disease in MPSII: preclinical evaluation of IDS-loaded PLGA nanoparticles. Int J Mol Sci. 2019;20:8. doi:10.3390/ijms20082014

13. Luk BT, Zhang L. Cell membrane-camouflaged nanoparticles for drug delivery. $J$ Control Release. 2015;220(Pt B):600-607. doi:10.1016/j.jconrel.2015.07.019

14. Zheng N, Liu W, Li B, et al. Co-delivery of sorafenib and metapristone encapsulated by CXCR4-targeted PLGA-PEG nanoparticles overcomes hepatocellular carcinoma resistance to sorafenib. $J$ Exp Clin Cancer Res. 2019;38(1):232. doi:10.1186/s13046-019-1216-x

15. Byeon Y, Lee JW, Choi WS, et al. CD44-targeting PLGA nanoparticles incorporating paclitaxel and FAK siRNA overcome chemoresistance in epithelial ovarian cancer. Cancer Res. 2018;78(21):6247-6256.

16. Dai W, Wang X, Song G, et al. Combination antitumor therapy with targeted dual-nanomedicines. Adv Drug Deliv Rev. 2017;115:23-45. doi:10.1016/j.addr.2017.03.001

17. Li R, He Y, Zhang S, Qin J, Wang J. Cell membrane-based nanoparticles: a new biomimetic platform for tumor diagnosis and treatment. Acta Pharm Sin B. 2018;8(1):14-22. doi:10.1016/j.apsb.2017.11.009

18. An FF, Zhang XH. Strategies for preparing albumin-based nanoparticles for multifunctional bioimaging and drug delivery. Theranostics. 2017;7(15):3667-3689. doi:10.7150/thno.19365

19. Kroll AV, Fang RH, Zhang L. Biointerfacing and applications of cell membrane-coated nanoparticles. Bioconjug Chem. 2017;28(1):23-32. doi:10.1021/acs.bioconjchem.6b00569

20. Luan X, Sansanaphongpricha K, Myers I, Chen H, Yuan H, Sun D. Engineering exosomes as refined biological nanoplatforms for drug delivery. Acta Pharmacol Sin. 2017;38(6):754-763.

21. Li SP, Lin ZX, Jiang XY, Yu XY. Exosomal cargo-loading and synthetic exosome-mimics as potential therapeutic tools. Acta Pharmacol Sin. 2018;39(4):542-551. doi:10.1038/aps.2017.178

22. Khodabandehlou K, Masehi-Lano JJ, Poon C, Wang J, Chung EJ. Targeting cell adhesion molecules with nanoparticles using in vivo and flow-based in vitro models of atherosclerosis. Exp Biol Med (Maywood). 2017;242(8):799-812. doi:10.1177/1535370217693116

23. Wang $\mathrm{H}$, Wu J, Williams GR, et al. Platelet-membrane-biomimetic nanoparticles for targeted antitumor drug delivery. J Nanobiotechnology. 2019;17(1):60. doi:10.1186/s12951-019-0494-y

24. de la Torre P, Pérez-Lorenzo MJ, Alcázar-Garrido Á, Flores AI. Cellbased nanoparticles delivery systems for targeted cancer therapy: lessons from anti-angiogenesis treatments. Molecules. 2020;25:3. doi: $10.3390 /$ molecules 25030715

25. Fang RH, Jiang Y, Fang JC, Zhang L. Cell membrane-derived nanomaterials for biomedical applications. Biomaterials. 2017;128:69-83. doi:10.1016/j.biomaterials.2017.02.041

26. Wu M, Le W, Mei T, et al. Cell membrane camouflaged nanoparticles: a new biomimetic platform for cancer photothermal therapy. Int J Nanomedicine. 2019;14:4431-4448. doi:10.2147/IJN.S200284

27. Xia Q, Zhang Y, Li Z, Hou X, Feng N. Red blood cell membrane-camouflaged nanoparticles: a novel drug delivery system for antitumor application. Acta Pharm Sin B. 2019;9(4):675-689. doi:10.1016/j.apsb.2019.01.011

28. Sun Y, Su J, Liu G, et al. Advances of blood cell-based drug delivery systems. Eur $J$ Pharm Sci. 2017;96:115-128. doi:10.1016/j. ejps.2016.07.021

29. Chen H, Sha H, Zhang L, et al. Lipid insertion enables targeted functionalization of paclitaxel-loaded erythrocyte membrane nanosystem by tumor-penetrating bispecific recombinant protein. Int J Nanomedicine. 2018;13:5347-5359. doi:10.2147/IJN.S165109
30. Wang D, Dong H, Li M, et al. Erythrocyte-cancer hybrid membrane camouflaged hollow copper sulfide nanoparticles for prolonged circulation life and homotypic-targeting photothermal/chemotherapy of melanoma. ACS Nano. 2018;12(6):5241-5252. doi:10.1021/ acsnano. $7 \mathrm{~b} 08355$

31. Liu W, Ruan M, Wang Y, et al. Light-triggered biomimetic nanoerythrocyte for tumor-targeted lung metastatic combination therapy of malignant melanoma. Small. 2018;14(38):e1801754. doi:10.1002/ smll.201801754

32. Yang Q, Xiao Y, Yin Y, Li G, Peng J. Erythrocyte membrane-camouflaged IR780 and DTX coloading polymeric nanoparticles for imaging-guided cancer photo-chemo combination therapy. Mol Pharm. 2019;16(7):3208-3220. doi:10.1021/acs. molpharmaceut.9b00413

33. Lavergne M, Janus-Bell E, Schaff M, Gachet C, Mangin PH. Platelet integrins in tumor metastasis: do they represent a therapeutic target? Cancers (Basel). 2017;9:10. doi:10.3390/cancers9100133

34. Zhai Y, Su J, Ran W, et al. Preparation and application of cell membrane-camouflaged nanoparticles for cancer therapy. Theranostics. 2017;7(10):2575-2592. doi:10.7150/thno.20118

35. Hu CM, Fang RH, Wang KC, et al. Nanoparticle biointerfacing by platelet membrane cloaking. Nature. 2015;526(7571):118-121. doi:10.1038/nature 15373

36. Xu P, Zuo H, Chen B, et al. Doxorubicin-loaded platelets as a smart drug delivery system: an improved therapy for lymphoma. Sci Rep. 2017;7:42632. doi:10.1038/srep42632

37. Rao L, Bu LL, Ma L, et al. Platelet-facilitated photothermal therapy of head and neck squamous cell carcinoma. Angew Chem Int Ed Engl. 2018;57(4):986-991. doi:10.1002/anie.201709457

38. Shang Y, Wang Q, Li J, et al. Platelet-membrane-camouflaged zirconia nanoparticles inhibit the invasion and metastasis of hela cells. Front Chem. 2020;8:377. doi:10.3389/fchem.2020.00377

39. Deng G, Sun Z, Li S, et al. Cell-membrane immunotherapy based on natural killer cell membrane coated nanoparticles for the effective inhibition of primary and abscopal tumor growth. ACS Nano. 2018;12(12):12096-12108. doi:10.1021/acsnano.8b05292

40. Pang L, Qin J, Han L, et al. Exploiting macrophages as targeted carrier to guide nanoparticles into glioma. Oncotarget. 2016;7 (24):37081-37091. doi:10.18632/oncotarget.9464

41. Xuan M, Shao J, Dai L, He Q, Li J. Macrophage cell membrane camouflaged mesoporous silica nanocapsules for in vivo cancer therapy. Adv Healthc Mater. 2015;4(11):1645-1652. doi:10.1002/ adhm.201500129

42. Zhang Y, Cai K, Li C, et al. Macrophage-membrane-coated nanoparticles for tumor-targeted chemotherapy. Nano Lett. 2018;18 (3):1908-1915. doi:10.1021/acs.nanolett.7b05263

43. Batrakova EV, Kim MS. Using exosomes, naturally-equipped nanocarriers, for drug delivery. J Control Release. 2015;219:396-405. doi:10.1016/j.jconrel.2015.07.030

44. Kalluri R, LeBleu VS. The biology, function, and biomedical applications of exosomes. Science. 2020;367:6478. doi:10.1126/science.aau6977

45. Yong T, Zhang X, Bie N, et al. Tumor exosome-based nanoparticles are efficient drug carriers for chemotherapy. Nat Commun. 2019;10 (1):3838.

46. Jia Y, Chen Y, Wang Q, et al. Exosome: emerging biomarker in breast cancer. Oncotarget. 2017;8(25):41717-41733. doi:10.18632/oncotarget. 16684

47. Fitts CA, Ji N, Li Y, Tan C. Exploiting exosomes in cancer liquid biopsies and drug delivery. Adv Healthc Mater. 2019;8(6):e1801268. doi:10.1002/adhm.201801268

48. Liao W, Du Y, Zhang C, et al. Exosomes: the next generation of endogenous nanomaterials for advanced drug delivery and therapy. Acta Biomater. 2019;86:1-14. doi:10.1016/j.actbio.2018.12.045

49. Shao H, Im H, Castro CM, Breakefield X, Weissleder R, Lee H. New technologies for analysis of extracellular vesicles. Chem Rev. 2018;118(4):1917-1950. doi:10.1021/acs.chemrev.7b00534 
50. Kamerkar S, LeBleu VS, Sugimoto H, et al. Exosomes facilitate therapeutic targeting of oncogenic KRAS in pancreatic cancer. Nature. 2017;546(7659):498-503.

51. Huang X, Wan F, Ma L, et al. Investigation of copper-cysteamine nanoparticles as a new photosensitizer for anti-hepatocellular carcinoma. Cancer Biol Ther. 2019;20(6):812-825. doi:10.1080/ 15384047.2018.1564568

52. Wei H, Chen J, Wang S, et al. A nanodrug consisting of doxorubicin and exosome derived from mesenchymal stem cells for osteosarcoma treatment in vitro. Int $J$ Nanomedicine. 2019;14:8603-8610. doi:10.2147/IJN.S218988
53. Sancho-Albero M, Navascués N, Mendoza G, et al. Exosome origin determines cell targeting and the transfer of therapeutic nanoparticles towards target cells. J Nanobiotechnology. 2019;17(1):16.

54. Morishita M, Takahashi Y, Matsumoto A, Nishikawa M, Takakura Y. Exosome-based tumor antigens-adjuvant co-delivery utilizing genetically engineered tumor cell-derived exosomes with immunostimulatory CpG DNA. Biomaterials. 2016;111:55-65. doi:10.1016/j. biomaterials.2016.09.031

\section{Publish your work in this journal}

Drug Design, Development and Therapy is an international, peerreviewed open-access journal that spans the spectrum of drug design and development through to clinical applications. Clinical outcomes, patient safety, and programs for the development and effective, safe, and sustained use of medicines are a feature of the journal, which has also been accepted for indexing on PubMed Central. The manuscript management system is completely online and includes a very quick and fair peer-review system, which is all easy to use. Visit http://www. dovepress.com/testimonials.php to read real quotes from published authors. 